\title{
Multifocal Longitudinally Extensive Transverse Myelitis as a Rare Complication of Mumps Infection
}

\author{
So Hyun Ahn ${ }^{*}$, , Jae-Seok Kim ${ }^{\ddagger}$, Jong Seok Bae ${ }^{\dagger}$, Yerim Kim ${ }^{\dagger}$ \\ Department of Neurology, Seoul National University Hospital ${ }^{\star}$, Seoul; Department of Neurology, Kangdong Sacred Heart Hospital, Hallym \\ University College of Medicine ${ }^{\dagger}$, Seoul; Department of Laboratory Medicine, Kangdong Sacred Heart Hospital, Hallym University College of \\ Medicine ${ }^{\ddagger}$, Seoul, Korea
}

Myelitis is one of the rare neurological complications of mumps infection. However, there have been few reports of multifocal longitudinally extensive transverse myelitis (LETM) as a complication during the convalescent stage after acute mumps infections. A 14-year-old boy abruptly developed paraplegia, paresthesia of both legs, and urinary retention 4 weeks after acute mumps infection, from which he had recovered with supportive treatment. Spinal magnetic resonance imaging revealed two separate LETMs lesions over the thoracic spinal cord. Other radiological and electrophysiological studies revealed no evidence of demyelinating disorders. The results of laboratory studies were all negative except for evidence of recent mumps infection. He was treated with a high-dose intravenous steroid, and recovered from his neurological symptoms about 1 month later. This is the first report of multifocal LETM after acute mumps infection. LETM is thus not only associated with neuroimmunological syndromes, such as neuromyelitis optica, but it can also develop after acute viral infection.

\section{J Neurosonol Neuroimag 201\$;10(1):37-40}

Key Words: Mumps; Myelitis; Transverse; Complications; Paraplegia
Received: April 25, 2018

Revised: May 21, 2018

Accepted: May 24, 2018

Address for correspondence: Yerim Kim

Department of Neurology, Kangdong Sacred Heart Hospital, Hallym University College of Medicine, 150 Seongan-ro, Gangdong-gu, Seoul 05355, Korea

Tel: $+82-2-2224-2206$

Fax: $+82-2-487-6330$

E-mail: brainyrk@gmail.com
Longitudinally extensive transverse myelitis (LETM) is defined as a myelitis that longitudinally extends over at least three serially adjacent vertebral segments. LETM is associated with several conditions, with neuromyelitis optica (NMO) being the most frequent, followed by spinal cord infarction and para- or postinfectious myelopathy, involving various pathogens. ${ }^{1}$

Acute mumps infection is known to be associated with various neurological complications, such as aseptic meningitis, acute encephalitis, chronic encephalitis, hydrocephalus, cerebellar ataxia, arthritis, orchitis, presternal edema, transverse myelitis, Guillain-Barré syndrome, and deafness. ${ }^{2}$ Of these, transverse myelitis has been reported as a rare complication of this infection. Previously reported cases of transverse myelitis after mumps infection were complicated with encephalitis, and were more common in younger children. Most of the cases had a slow recovery from the myelitis. ${ }^{3,4}$
Here we report a boy who presented with paraparesis and urinary retention due to an LETM as a rare neurological complication after mumps infection.

\section{CASE REPORT}

A 14-year-old boy came to our department with a 3-day history of inability to walk and pain in both legs. He had a history of acute mumps infection 4 weeks prior, for which he had been treated at the pediatric clinic at our hospital. Since mumps infection, he had no other history of infection.

Physical examination revealed normal vital signs. There were no systemic signs suggesting acute or subacute mumps infections. The straight-leg raising test readily evoked a radiating pain over the bilateral lower extremities. Neurological examination revealed 
paraparesis during manual motor power examination. Hip flexion/extension and knee flexion/extension were reduced to Medical Research Council (MRC) grade 2/2, and ankle dorsiflexion, plantar flexion, and foot inversion/eversion were reduced to MRC grade $1 / 1$. The deep-tendon reflexes on the knee and ankle were absent, and there was hyperesthesia and allodynia below the T6 level. The patient had urinary retention and anal reflex tone was diminished. He was thought to suffer from spinal shock due to an acute cord lesion at around T6, for which emergent spine magnetic resonance imaging (MRI) was performed.

A T2-weighted thoracic spine MRI (Fig. 1) revealed hyperintensity lesion in $\mathrm{T}_{2}-5$ and $\mathrm{T}_{9-11}$ spinal cord with moderate swelling of the cord at these levels. Laboratory studies including complete blood count, and renal and liver function tests were normal. Cerebrospinal fluid (CSF) analysis revealed pleocytosis (a white blood cell count of $130 / \mathrm{mm}^{3}$ ) with elevated albumin concentration ( $38 \mathrm{mg} / \mathrm{dL}$; normal range 10-30), but otherwise was normal. Anti-mumps IgG and IgM tests in serum were positive (Platelia Mumps IgM, immunoenzymatic capture method and Platelia Mumps IgG enzyme-linked immunosorbent assay [ELISA]; Bio-Rad, Marnes-la-Coquette, France). Polymerase chain reaction (PCR) for mumps virus, Epstein-Barr virus (EBV), herpes simplex virus (HSV) 1, HSV2, varicella zoster virus (VZV), and enterovirus in CSF yielded negative
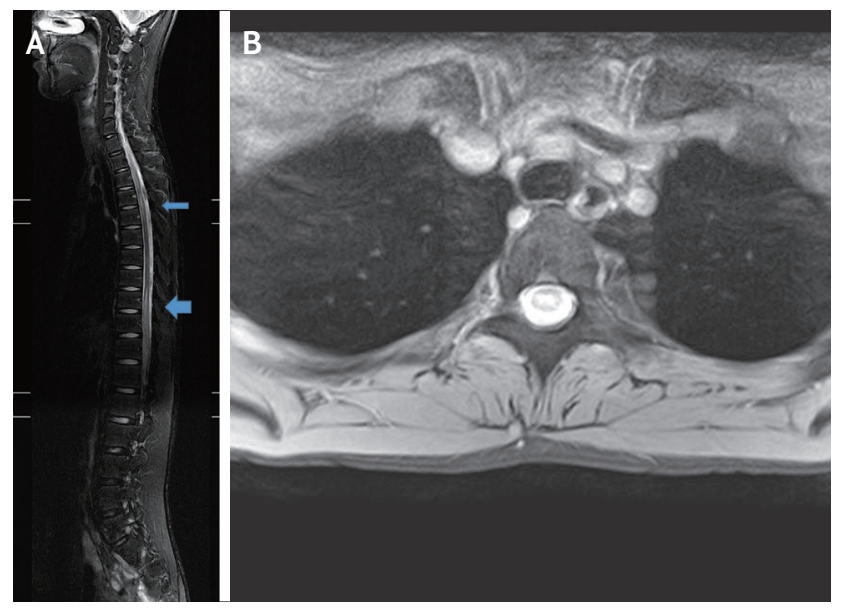

FIG. 1. A T2-weighted thoracic spine magnetic resonance imaging. (A) A T2-weighted sagittal image shows multisegmental longitudinal, high-signal-intensity lesions with cord swelling at T2-5 (thin arrow) and T9-11 (thick arrow). (B) Axial view of a T2-weighted image showing a centrally located lesion occupying more than half of the cord. results. Serum anti-aquaporin-4 (anti-AQP-4) antibody test was negative. CSF oligoclonal band analysed with concurrent serum sample and the result were negative. Visual evoked potential test showed no conduction abnormalities. In addition, brain MRI showed neither a pathological lesion nor an abnormally enhancing pattern. In total, other relapsing demyelinating disorders, such as NMO or multiple sclerosis were excluded. To exclude other autoimmune diseases, we evaluated autoimmune antibodies in serum. Rheumatoid factor, c-antineutrophil cytoplasmic antibodies (ANCA), p-AN$\mathrm{CA}$, anti-ds DNA, and paraneoplastic antibodies including anti-Hu, anti-Yo, and anti-Ri were negative results. Although fluorescent antinuclear antibody (FANA) was positive (titer 1:140, speckled pattern), it did not have clinical significance due to negative anti-extractable nuclear antigen (ENA) panel.

The patient was finally diagnosed as having post- or parainfectious LETM following mumps infection and he was treated with intravenous methylprednisolone ( $1 \mathrm{~g} /$ day for 5 days). A comprehensive rehabilitation program was also prescribed. During the course of treatment the patient exhibited marked improvement of weakness by the $5^{\text {th }}$ day of admission, and on the 7 th day began to walk using walking aid. His urinary retention also improved gradually. He had recovered fully from the motor weakness and urinary symptoms 6 weeks after symptom onset. In next 6 months, he showed no suspicious symptoms of relapse.

\section{DISCUSSION}

Most of the previous reports on the neurological complications after mumps infection have described disseminated syndromes over the entire central nervous system, such as meningoencephalitis or encephalomyelitis. ${ }^{4,5}$ The present case differs from these reports in that our patient presented with only paraparesis and urinary difficulty due to acute LETM. In addition, his recovery from the neurological impairment was considerably faster than has been reported previously.

LETM can be associated with several conditions, of which NMO is the most common, followed by spinal cord infarction and para- or postinfectious myelopathy. NMO is a relapsing-remitting inflammatory disease 
that preferentially involves the optic nerves and spinal cord. Anti-AQP-4 antibodies are highly specific for NMO and its spectrum disorders, and are considered to play a central role in the immunopathogenesis of the disorder. Therefore, both NMO and NMO-spectrum disease can be diagnosed using commercially available antibodies to AQP-4. ${ }^{1,6}$

Interestingly, laboratory evidence of previous viral infection has been reported in some cases of NMO (e.g., mumps, measles, rubella, human cytomegalovirus, VZV, EBV, hepatitis A virus, human immunodeficiency virus, and dengue virus). ${ }^{7}$ In this context, some authors claimed that these infectious pathogens are either directly involved or activate a major immunological step in the pathogenesis of NMO. This suggestion raises the need to evaluate NMO or NMO-spectrum disease in the setting of para- or postinfectious myelopathy.

However, AQP-4 antibody testing in cases of mumps-related myelitis has not been reported previously. Whether or not NMO or NMO-spectrum disease can initially present with para- or postinfectious myelopathy has yet to be established.

The patient described herein presented with both serological evidence of mumps infection and previous typical symptoms of mumps infection, and the observed neurological involvement was not disseminated like in previous reports. Therefore, although this patient exhibited only LETM without optic neuritis or brain lesions, the possibility of NMO or NMO-spectrum disease as a cause of the LETM was suspected. However, both CSF analysis with RNA-PCR for mumps and serum testing for antibodies to AQP4 yielded negative findings. This case does not meet the "2017 McDonald criteria" or the "2015 International consensus diagnostic criteria for neuromyelitis optica spectrum disorders". ${ }^{9}$ However, we cannot fully exclude the first attack of other demyelinating disease, such as a 'clinically isolated syndrome (CIS)'. Of further relevance, it is more likely to be post-infectious myelitis due to myelitis occurring after a certain period of mumps infection. Although the sensitivity of reverse transcriptase polymerase chain reaction (RT-PCR) was approximately $70 \%$, the time of sampling is critical for the diagnosis of mumps virus infection in $\mathrm{CSF}^{10}$ Therefore, we concluded that this patient's diagnosis was postinfectious LETM as a rare complication of mumps. Despite its rare occurrence, the findings of this case report suggest that acute mumps infection can be complicated by postinfectious LETM, mimicking NMO or NMO-spectrum disease. Clinicians need to be familiar with this rare complication and its differential diagnosis.

\section{Acknowledgments}

This work was supported by Basic Science Research Program through the National Research Foundation of Korea (NRF) funded by the Ministry of Education under Grant (NRF-2017R1D1A1B03029672).

\section{Conflicts of Interest}

All authors have no conflicts of interest.

\section{REFERENCES}

1. Tobin WO, Weinshenker BG, Lucchinetti CF. Longitudinally extensive transverse myelitis. Curr Opin Neurol. 2014;27:279-289.

2. Caksen H, Ustünbaş HB. A fatal case of acute transverse myelitis associated with mumps. J Emerg Med. 2003;24:341342.

3. Savaş L, Arlier Z, Akçali A, Karataş M, Onlen Y. Full recovered meningoencephalomyelitis caused by mumps virus. Eur J Neurol. 2004;11:639-640.

4. Unal A, Emre U, Atasoy HT, Sumer MM, Mahmutyazicioglu K. Encephalomyelitis following mumps. Spinal Cord. 2005;43:441-444.

5. Sonmez FM, Odemis E, Ahmetoglu A, Ayvaz A. Brainstem encephalitis and acute disseminated encephalomyelitis following mumps. Pediatr Neurol. 2004;30:132-134.

6. Kitley J, Leite MI, Küker W, Quaghebeur G, George J, Waters $\mathrm{P}$, et al. Longitudinally extensive transverse myelitis with and without aquaporin 4 antibodies. JAMA Neurol. 2013;70:1375-1381.

7. Koga M, Takahashi T, Kawai M, Fujihara K, Kanda T. A serological analysis of viral and bacterial infections associated with neuromyelitis optica. J Neurol Sci. 2011;300:19-22.

8. hompson AJ, Banwell BL, Barkhof F, Carroll WM, Coetzee T, Comi G, et al. Diagnosis of multiple sclerosis: 2017 revisions of the McDonald criteria. Lancet Neurol. 2018;17:162-173.

9. Wingerchuk DM, Banwell B, Bennett JL, Cabre P, Carroll W, Chitnis T, et al. International consensus diagnostic criteria 
So Hyun Ahn, et al. Multifocal LETM after Mumps

for neuromyelitis optica spectrum disorders. Neurology. 2015;85:177-189.

10. Uchida K, Shinohara M, Shimada S, Segawa Y, Doi R, Gotoh
A, et al. Rapid and sensitive detection of mumps virus RNA directly from clinical samples by real-time PCR. J Med Virol. 2005;75:470-474. 